\title{
Immunotherapy of advanced thyroid cancer: from bench to bedside
}

\author{
Sonia Moretti, Elisa Menicali, Nicole Nucci, Martina Guzzetti, Silvia Morelli and \\ Efisio Puxeddu (D)
}

Department of Medicine, University of Perugia, Perugia, Italy
Correspondence

should be addressed

to E Puxeddu

Email

efisio.puxeddu@unipg.it

\begin{abstract}
Immunotherapy has arisen in use in the field of oncology with seven immune checkpoint inhibitors approved for the treatment of a variety of cancer histologies. Depending on the cancer type, the success rate might be different, but in average it is about $20 \%$, with some cases showing a durable response, lasting also after the interruption of the treatment, with a clear benefit on OS. The development of an efficacious cure for advanced thyroid carcinomas is still an unmet need and immunotherapy represents an interesting alternative option also for this cancer. However, very few clinical trials have been accomplished and very few studies exploring a way to overcome resistance have been performed. In this review, we will summarize the mechanisms of immune escape, with a special reference to follicularderived thyroid carcinoma. Furthermore, we will try to speculate on the use of immune checkpoint inhibitors for the treatment of follicular-derived advanced thyroid carcinoma. Finally, we will summarize the ongoing clinical trials and the future directions of the field.
\end{abstract}

\section{Introduction}

In 2011, Hanahan and Weinberg included tumor capacity to avoid immune destruction among the hallmarks of cancer (1). In detail, transformed cells appeared to express neoantigens that are able to break self-tolerance and to induce an anti-tumor immune reaction (2). Simultaneously, the immune system appeared to provide a very efficient immune surveillance apparatus that recognizes and eliminates the transformed cells (Elimination) (3). In this way the immune system restrains the development of cancers (3). However, few transformed cells that resist immune surveillance may develop. Those cells enter an immunoediting process and finally gain the capacity to escape the immune system (3).

The knowledge that the immune system can be reactivated against cancer cells and used to cure the disease dates back to the end of the nineteenth century, when seminal work by William Cooley demonstrated that bacterial infections induced in cancer patients could

\section{Invited Author's profile}

Prof. Efisio Puxeddu has been working, since 2007, at the Division of Internal Medicine and Endocrine and Metabolic Sciences of the University of Perugia, first as Assistant Professor and then from 2015 as Associate Professor of Endocrinology. From 2002 to 2009 he worked on the role of BRAF mutations in papillary thyroid carcinoma development and prognosis. Since 2010, his research has focused on the study of thyroid immuno-oncology.

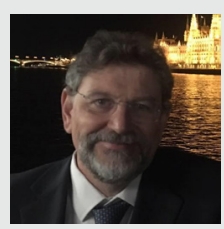


eliminate or at least slow down the disease (4). However, for several decades the interest for immune-oncology stayed at the margins of anti-cancer research. Things changed drastically 10 years ago, thanks to the seminal work of the two 2018 Nobel Price winners for Medicine, James Patrick Allison and Tasuku Honjo. In detail, Dr Allison's work demonstrated the importance of lymphocyte inhibitory receptor CTLA4 in preventing the co-stimulation of T cells during the lymph node priming phase of the immune response (5). Subsequent blockade of CTLA4 by using anti-CTLA4 monoclonal antibodies allowed to obtain tumor rejection and represented the proof-of-concept for the use of immune checkpoint inhibitors in cancer treatment (6). Similarly, Dr Honjo's work demonstrated that the interaction of the T-cell receptor PD1 with its ligands PDL1 and PDL2 inhibits the effector phase of T-cell activation and that cancers overexpress these PD1 ligands (7). Also, in this case, blockade of PD1-PDL1 interaction by using monoclonal antibodies against the receptor or its ligand showed anti-tumor effects (8).

As a consequence of those important findings as many as seven immune checkpoint inhibitors have received FDA approval for cancer treatment in the last 10 years: The CTLA4 inhibitor ipilimumab for the treatment of melanoma (9); the two PD1 inhibitors nivolumab and pembrolizumab for the treatment of melanoma, NSCLC, renal cell carcinoma, head and neck squamous cell carcinoma, urothelial carcinoma, and microsatellite instability-high colorectal cancer, among several other tumor types (9); the novel PD1 inhibitor cemiplimab for the treatment of metastatic cutaneous squamous cell carcinoma (9); and the PDL1 inhibitors atezolizumab, avelumab, and durvalumab for the treatment of many of the same histologies as the PD1 inhibitors (9).

Life-threatening traditional therapy-resistant aggressive thyroid carcinomas are a minority with an incidence estimated to be $4-5$ per 1000000 men and women per year (10). However, the latter are the real clinical challenge. The introduction of targeted therapies with tyrosine kinase inhibitors (TKIs) (i.e. lenvatinib or sorafenib for differentiated thyroid carcinoma (DTC), dabrafenib plus trametinib for anaplastic thyroid carcinoma (ATC)) changed the natural history of those cancers. Unfortunately, lack of strong cytotoxic action, fast development of resistance and occurrence of side effects limit TKIs efficacy (11). Thus, novel therapeutic approaches are needed for aggressive thyroid cancers, including immunotherapy.

In this review, we will summarize the mechanisms of immune escape, with a special reference to follicular- derived thyroid carcinoma. Furthermore, we will try to speculate on the use of immune checkpoint inhibitors (ICI) for the treatment of follicular-derived advanced thyroid carcinoma. Finally, we will summarize the ongoing clinical trials and the future directions of the field.

\section{Immune escape in thyroid carcinoma}

Immune escape represents the last step of a process that starts with the development of transformed cells that are resistant to immune surveillance (Equilibrium) and progresses through the Immunoediting phase where, due to genetic or epigenetic changes in the transformed cell, but above all to modifications of the tumor microenvironment (TME) and of the interactions between the tumor cells and the TME, the developing cancer acquires an increasing refractoriness to the restraining effect of the immune system (3). Several mechanisms play a role in this process and in the realization of the final immune escape.

\section{Antigen presentation}

Transformed cells express neoantigens that may break self-tolerance. These antigens include tumor-specific mutated antigens represented by driver or passenger gene mutations and gene rearrangements and tumorassociated antigens that derive from gene overexpression, expression of embryonic or viral proteins, expression of tissue restricted differentiation antigens, and alternative glycosylation. These neoantigens are processed inside the cell and the resulting epitopes are exposed at its surface in association with molecules of the major histocompatibility complex class I (MHC-I) (2). Thus, tumor immunogenicity and anti-tumor immunity primarily depend on tumor mutational burden (TMB) and neoantigen load (12). Poorly immunogenic tumors with low $\mathrm{TMB}$, that is, pancreatic or prostate cancer, have a low infiltration of adaptive immune cells (12). Genetic instability due to alterations in DNA repair and replication genes can increase immunogenicity, inducing a high mutational burden and neoantigen load. An important example is given by those tumors across a wide range of histologies that are characterized by deficiencies in the DNA mismatch repair that lead to microsatellite instability (13).

In tumor cells, antigen presentation and T-cell activation may be impaired by recruitment of suppressed dendritic cells (DCs). This phenomenon may be caused 
by overexpression of $\beta$-catenin that mediates a decreased expression of the cytokine CCL4 and inability to recruit CD103+ DCs (see also 'Classic Oncologic Pathways') (14) or by other functional defects of the DC themselves that impair their ability to prime anti-tumor immune responses (15) (Fig. 1A).

Furthermore, reduced antigen presentation and T-cell activation may depend at the tumor level on downregulation of antigen or MHC-I expression (9), loss of function of $\beta 2$-microglobulin with disruption of MHC-I folding and transport to the cell surface (i.e. truncating mutations) (9), and mutations within the T-cell receptor binding domain of MHC-I (9) (Fig. 1A).

Interestingly, Angell and coworkers demonstrated that both MHC class I and $\beta 2$-microglobulin expression were reduced or absent in $76 \%$ of papillary thyroid carcinoma (PTC) specimens and were associated with reduced tumor-infiltrating immune cells, including

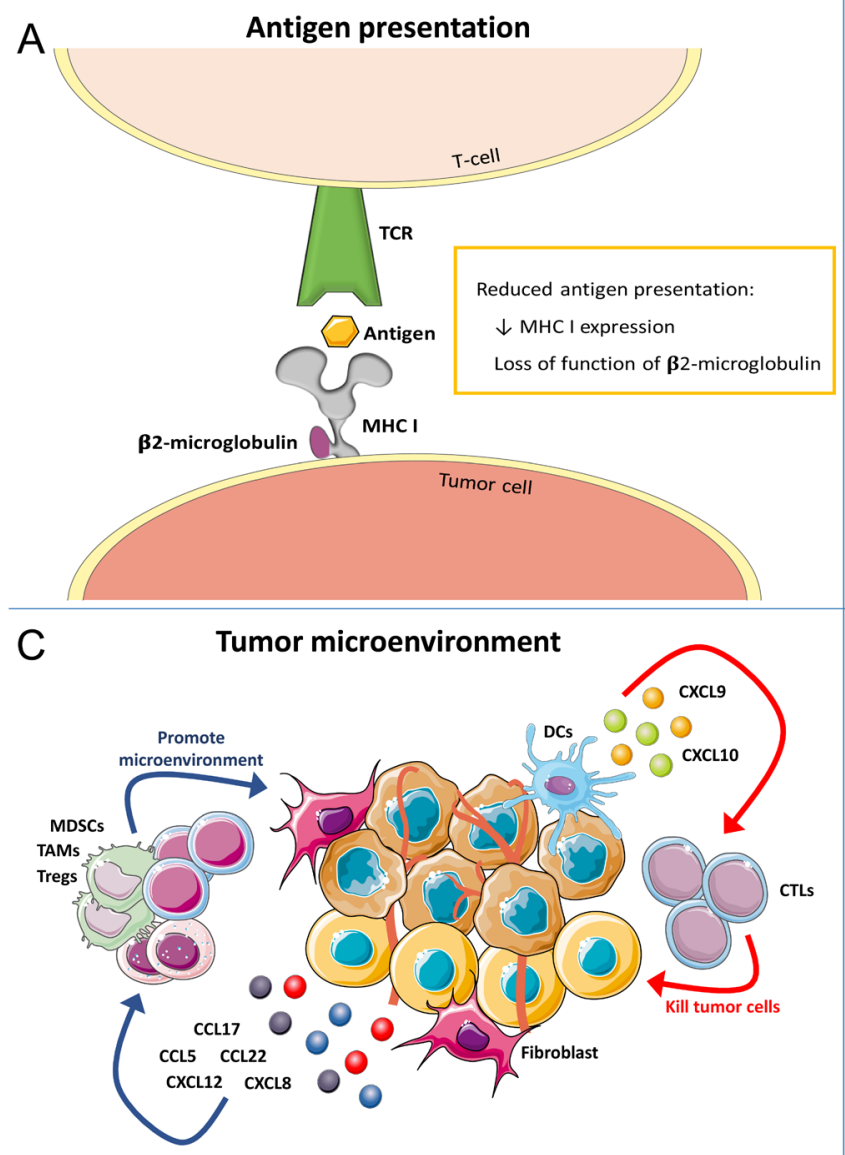

B Immune checkpoints

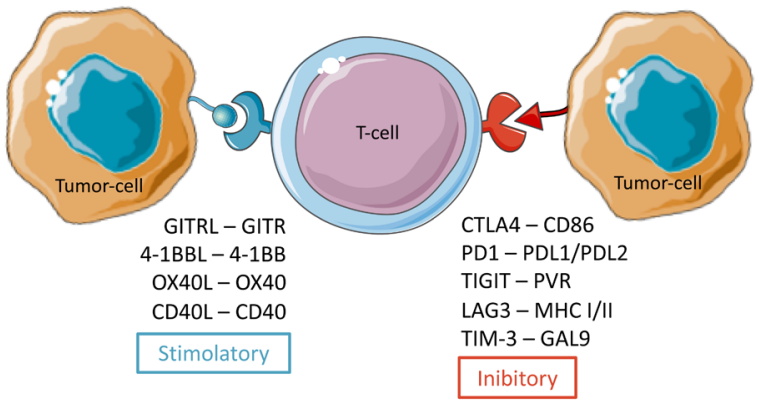

$\mathrm{D}$ Classic oncologic pathway

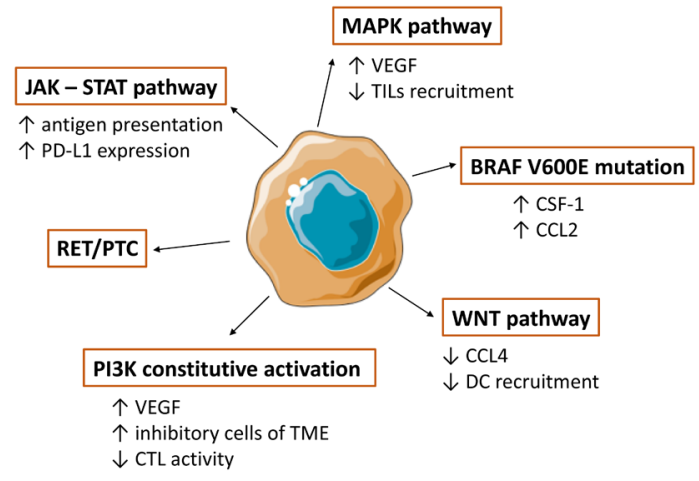

\section{Figure 1}

Immune escape in thyroid carcinoma. (A) Antigen presentation. In tumor cells, antigen presentation and T-cell activation may be impaired by recruitment of suppressed and inefficient DC, down-regulation of antigen, MHC-I or $\beta 2$-microglobulin expression. (B) Immune checkpoints. There is a great redundancy of immune checkpoints able to inhibit or activate the immune response. ATC, but not PDTC, showed up-regulation of both inhibitory and stimulatory immune checkpoint mediators. (C) Tumor microenvironment. Evolution of cancer is strongly dependent on the tumor microenvironment (TME) in which it develops. The TME comprises a variety of cell types including fibroblasts, endothelial cells, blood vessels, lymph vessels, CTLs, Tregs, TAMs, MDSCs, TAMCs, and DCs. The TME is regulated by several soluble factors (cytokines, chemokines, and products of cellular metabolism). High expression of CXCL9 and CXCL10 chemokines stimulates the recruitment of CTLs that kill the tumor cells and restrain cancer growth. Conversely, high expression of CCL5, CCL17, CCL22, CXCL8, and CXCL12 stimulates infiltration of MDSCS, TAMs, and Tregs that promote an immunosuppressive microenvironment. (D) Classic oncologic pathways. Several oncologic pathways might alter immune cell composition and cytokine profile of cancers and contribute to immune escape. The major effects on TME of the principal oncogenes involved in thyroid carcinogenesis are described. 
effector and suppressor populations (16). These data indicate that MHC-I down-regulation may play a role in immune escape in a subgroup of thyroid cancers.

Finally, also loss of IFN $\gamma$-induced JAK/STAT signaling may result in the inability to up-regulate MHC-I (and PDL1), resulting in reduced antigen presentation (9).

\section{Immune checkpoints}

Immune checkpoints are molecular switches able to inhibit or activate the immune response. Most of them are based on the expression of receptors on the surface of T-lymphocytes and other immune cells whose engagement by cognate ligands modulates proliferation and activation of the harboring cells. There is an enormous redundancy of these systems. In detail, among inhibitory immune checkpoints, CTLA4 with CD80 and CD86, PD1 with PDL1 and PDL2, TIGIT with PVR, TIM-3 with Galectin 9, and BTLA with HVEM, LAG3 and VISTA can be mentioned (9) (Fig. 1B). Furthermore, among stimulatory checkpoints, GITR with GITRL, 4-1BB with 4-1BBL, CD40L with CD40, ICOS with B7-H2, and OX40 with OX40L should be quoted (9) (Fig. 1B). CTLA4 and PD1 are the most frequently expressed receptors. In detail, CTLA4 is expressed on the surface of lymphocytes during the lymph node priming phase after the binding of the TCR to the cognate epitope coupled with MHC class II molecules expressed on the surface of the antigen presenting cells. CTLA4 competes with CD28 for the binding of CD80 and CD86 and thus sequesters these ligands from the CD28 co-stimulatory signal. In this way, CTLA4 down-regulates the lymphocyte priming and eventually triggers clonal deletion (5). Conversely, PD1 is expressed on the surface of cytotoxic T-lymphocytes (CTL) during the peripheral effector phase after engagement of the lymphocytes with the cognate antigens. Binding of PD1 with PDL1 or PDL2, that are over-expressed at the surface of the target cells after the release of IFN $\gamma$ by the activated lymphocytes, inhibits the effector function of CTL, thus dampening the immune response (7). Tumors hijack this mechanism overexpressing PDL1 or PDL2 (7). Thus, activation of these inhibitory immune checkpoints can favor cancer immune escape. In the contest of cancer, blockade of CTLA4 hyper-activates an anti-tumor immune response, while blockade of PD1 or its ligands allows to reverse the immunosuppression in the TME (6). The other inhibitory immune checkpoints work in a similar manner. Conversely, engagement of stimulatory receptors expressed on the surface of lymphocytes by the cognate ligands triggers proliferation and functional activation of the harboring cells.
Several studies have investigated the expression of PDL1 in thyroid carcinoma (17). Most of the studies analyzed protein expression by immunohistochemistry (IHC) (17), while a few analyzed both protein and mRNA expression (17). Almost all confirmed overexpression of PDL1 both in differentiated and anaplastic carcinomas. A few showed a significant higher prevalence of positive cases in more advanced tumors (17). However, there appear to be some inconsistencies associated to differences in the antibodies used for the IHC and in the mRNA/protein correlations.

Very recently, Giannini and coworkers showed up-regulation of inhibitory immune checkpoint mediators CD86 and CTLA4, PDL1/PDL2 and PD1, PVR and TIGIT, LAG3 and TIM-3 and stimulatory immune checkpoint mediators GITRL and GITR, 4-1BB, OX40L and OX40, and CD40, mainly in ATCs and at a lower extent in a subgroup of PTCs. Conversely poorly differentiated thyroid carcinomas (PDTCs) did not appear to express at all these immune checkpoint mediators (18) (see also 'Immune phenotypes of thyroid carcinoma').

\section{Tumor microenvironment}

Increasing evidence demonstrates that the evolution of cancer is strongly dependent on the complex tumor microenvironment (TME) in which it develops (9). The TME comprises a variety of cellular entities including fibroblasts, endothelial cells, blood vessels, lymph vessels, and cells of the immune system such as among the more relevant CTLs, regulatory T cells (Tregs), tumor-associated macrophages (TAMs), myeloid-derived suppressive cells (MDSCs), tumor-associated mast cells (TAMCs), and DCs (9). In addition to the physical components within the tumor, the TME includes several soluble factors such as cytokines, chemokines, and products of cellular metabolism (9). High expression of CXCL9 and CXCL10 chemokines stimulates the recruitment of CTLs that kill the tumor cells and restrain cancer growth (9) (Fig. 1C). Indeed, adaptive immune cell infiltration has been shown to have a prognostic value superior to the classic tumor invasion criteria, including grade, stage, and metastatic status (9). Conversely, high expression of CCL5, CCL17, CCL22, CXCL8, and CXCL12 stimulates infiltration of MDSCs, TAMs, and Tregs that promote an immunosuppressive microenvironment (9) (Fig. 1C). In recent time, a classification of the cancer immune contexture for colorectal cancers, based on the density of infiltrating $\mathrm{CD}^{+}$(total) and $\mathrm{CD}^{+}$(CTLs) lymphocytes, was proposed. It included four major immune coordination profiles: Hot, altered - immunosuppressed, 
altered - excluded and cold extensively reviewed by Galon and Bruni in 2019 (19). Modifications in the tumor microenvironment play a pivotal role in immune escape. In the case of tumors infiltrated by immune cells, immune escape may rely on local immune suppression due to selection of less immunogenic tumor cells or activation of inhibitory pathways. Conversely, in the case of a lack of immune infiltration, immune escape depends on the selection of tumor cells that prevent an immune interaction or aberrant tumor vasculature and stroma that impair the infiltration of immune cells (20).

The role of tumor-infiltrating lymphocytes (TILs) in PTC outcome has been controversial. Some papers highlight an association with a favorable prognosis, including improved disease-free and overall survival, either in adults or in children and young adults (21). Conversely, other papers underscore a possible association with a bad prognosis $(22,23)$. The deeper analysis published by French and coworkers in 2010 allows to understand the controversy. Indeed, in their study, T-cell subpopulations appeared to have a different impact on outcome with CD4(+) T-cell frequency correlating with tumor size, FoxP3(+) regulatory T-cell (Treg) frequency correlating with lymph node metastases, and CD8 to Treg ratio correlating inversely with tumor size. Thus, $\mathrm{CD} 8^{+} \mathrm{T}$ cells appeared to antagonize cancer development, while Tregs appeared to favor oncogenesis (22), and the relative distribution of these subpopulations might influence the cancer outcome. The protumorigenic role of Tregs in thyroid cancer was further confirmed by other studies (23, 24). Furthermore, exhausted $\mathrm{PD} 1^{+} \mathrm{CD} 8^{+} \mathrm{T}$ cells appeared to be another marker of immunosuppression and bad outcome $(23,24)$.

In regards of pro-tumoral TAMs, they appeared to be overrepresented in advanced differentiated thyroid carcinomas (DTCs) and ATCs where they predicted a poor survival (21). Interestingly, in ATC they appeared to form an interconnected cellular supportive network that may trap T cells in the tumor stroma (25).

High CD1a-positive dendritic cell density was associated with improved disease-free survival in PTC (21). Conversely, in PTC, immature plasmocytoid DC (pDC) density appeared to be correlated with the density of ICOS(+)Foxp3(+) Tregs that represent a strong predictor of disease progression. These data suggested that PTCderived pDCs may induce the differentiation of naive CD4(+) T cells into ICOS(+)Foxp3(+)Tregs and in this way contribute to immune escape (21).

Elevated preoperative levels of circulating MDSCs have been reported in thyroid cancer patients in comparison to controls affected by non-tumoral thyroid diseases (21). Furthermore, a correlation between the number of circulating MDSCs and tumor aggressiveness could be detected in DTCs (21).

TAMC density was higher in 95\% of PTCs than in control tissues and correlated with extrathyroidal extension of the tumors (21). In thyroid cancer-activated MCs, the release of CXCL8/interleukin (IL)-8 appeared to mediate epithelial-to-mesenchymal transition (EMT) and stemness features of thyroid cancer cells (21).

\section{Classic oncologic pathway}

Several signaling pathways that are activated by aberrations in oncogenes or oncosuppressors might alter immune cell composition and cytokine profile of cancers and contribute to immune escape (Fig. 1D). Among those, as already mentioned, the IFN $\gamma$-JAK-STAT pathway up-regulates both antigen presentation and PDL1 expression. Its loss reduces immunogenicity of tumors (9). The constitutive activation of the mitogenactivated protein kinase (MAPK) pathway up-regulates the expression of VEGF and other inhibitory cytokines and impairs TILs recruitment and function (26). Constitutive activation of the phosphatidylinositol 3-kinase (PI3K) pathway through the deletion of the phosphatase and tensin homolog (PTEN) or activating mutation of PI3KCA decreases CTL activity through the expression of VEGF and recruitment of inhibitory cells to the TME (27). Constitutive WNT signaling with stabilization of $\beta$-catenin decreases expression of cytokine CCL4 and impairs recruitment of $\mathrm{CD} 103^{+} \mathrm{DC}$ needed for T-cell priming (14).

Conditional activation of $\mathrm{BRAF}^{\mathrm{V} 600 \mathrm{E}}$ in murine thyroids was associated to an increased expression of the TAM chemoattractants CSF1 and CCL2. This was followed by the development of PTCs that were densely infiltrated with TAMs that express CSF1/R and CCR2. Targeting CCR2-expressing cells during BRAF-induction reduced TAM density and impaired PTC development. This strategy also induced smaller tumors, decreased proliferation and restored a thyroid follicular architecture in established PTCs (28).

Interestingly, data produced by Puxeddu and coworkers (29), and later confirmed by others (30), clearly showed in a well differentiated normal thyroid cell model, characterized by the inducible expression of the RET/PTC3 oncogene, known for its role in tumor initiation, that, in the early stages of cell transformation, genetic programs are triggered that are not only designed to intrinsically block cell proliferation (31), and possibly induce 
senescence (32), but also to enable cell immunogenicity in order to extrinsically stimulate immune surveillance. It is therefore intuitive that the progression of tumorigenesis may occur only after activation of other genetic programs that overcome these restraining mechanisms, whose emergence is favored by the selection barrier determined by the immune surveillance itself. One of those is represented by the activation of the immunosuppressant indoleamine 2,3-dioxygenase 1 (IDO1) pathway. Indeed, IDO1 expression resulted significantly higher in all thyroid carcinoma histotypes compared to normal thyroid. Moreover, in Hashimoto's thyroiditis-negative PTCs, IDO1 mRNA expression magnitude correlated with FoxP3+ Treg density in the TME (33). Furthermore, a direct link between IDO1 expression and the oncogenic activation of RET (RET/PTC rearrangements or activating mutation of RET) in thyroid carcinoma, mediated by STAT1, could be demonstrated (34). Finally, also an IDO1 downstream ligand-dependent transcription factor, AhR, known to be activated by kynurenine, the metabolite produced from tryptophan by IDO1, resulted up-regulated in all thyroid carcinoma histotypes compared to normal thyroid and appeared to potentiate the immunosuppressive climate of the TME (35). AhR expression appeared to be significantly regulated by a constitutively active MAPK pathway due to BRAF $^{\mathrm{V} 600 \mathrm{E}}(36)$.

\section{Immune phenotypes of thyroid carcinoma}

Characterization of the immune phenotype of cancers has gained interest for its potential immunotherapeutic implications. In this regard, two recent studies analyzed the immune expression profile of thyroid carcinomas $(18,37)$.

In the first work by $\mathrm{Na}$ and Choi, the relationship between differentiation and immune landscape and its potential immunotherapeutic implications in PTC were analyzed by means of mRNA transcriptome data obtained in The Cancer Genome Atlas (TCGA) (37). Their data showed that high immune cell type enrichment (Immunoscore) was related with low thyroid differentiation score (TDS) and the presence of $\mathrm{BRAF}^{\mathrm{V} 600 \mathrm{E}}$ mutation. Moreover, the enrichment scores of myeloid cells (dendritic cells, macrophages and mast cells), B-cells and Tregs were increased in PTCs with low TDS and $\mathrm{BRAF}^{\mathrm{V} 600 \mathrm{E}}$ mutation. Finally, in thyroid tumors with high Immunoscore, low TDS and $\mathrm{BRAF}^{\mathrm{V} 600 \mathrm{E}}$ mutation, immunosuppressive marker expression (CTLA4, PDL1, and HLA-G) was increased.
In the second work, Giannini and coworkers studied the immune expression profile in different thyroid carcinoma histotypes (PTCs, PDTCs and ATCs) with the Nanostring platform (18). Expression data suggested the existence of two main clusters: one characterized by lower expression of immune-related genes, including all PDTCs and some PTCs, and the other characterized by up-regulation of immune-related genes, including almost all ATCs and the remaining PTCs. The clustering did not depend on the cancers' genotype. Interestingly, also TMB did not differ significantly between ATCs and PDTCs. The expression levels of genes specific for immune cell populations indicated that ATCs were characterized by a microenvironment richly infiltrated by macrophages and $\mathrm{T}$ cell. Thus, ATCs demonstrated a hot or altered-immunosuppressed immune coordination profile. These tumors could benefit from therapies that combine inhibitory immune checkpoints blockade and activating immune checkpoints stimulation eventually coupled to CSF-1/R blockers to deplete TAMs. Conversely, PDTCs displayed a tumor microenvironment poorly infiltrated by immune cells, showing a cold or alteredexcluded immune coordination profile. In these cases, immunotherapy should in the first instance foresee an increase in the immune infiltration of the tumor microenvironment in order to activate an effective antitumor immune response.

\section{Immune checkpoint inhibitors and thyroid carcinoma}

\section{Immune checkpoint inhibitors}

The development of an efficacious cure for advanced thyroid carcinomas is still an unmet need and immunotherapy represents an interesting alternative option. To date, no immunotherapy has been approved for advanced thyroid carcinoma. However, preclinical studies, a few case reports and clinical trials, are starting to produce indications for their use.

As mentioned in the introduction, as many as seven ICIs have been approved for the treatment of several cancers thus far. Depending on the cancer type, the success rate might be different, but in average it is about $20 \%$, with some cases showing a durable response, lasting also after the interruption of the treatment, with a clear benefit on overall survival (OS) (38). The current question is why we do experience resistance to this type of treatments in the remaining $80 \%$ of the cancer patients and how this resistance can be overcome. In many cases, response rate 
appeared to be proportional to TMB, probably due to a higher load in neoantigens and a higher immunogenicity that attract more anti-tumor CTLs inside the neoplasm (12). Increased PDL1 expression and elevated numbers of TILs represent other predictive biomarkers (39). Interestingly, ATC appear to express those predictive biomarkers at higher levels (18).

Recently published results obtained in 22 patients affected by advanced PTCs or FTCs, included in the phase Ib KEYNOTE-028 trial conducted to evaluate safety and antitumor activity of pembrolizumab as monotherapy in advanced solid tumors, showed two partial responses, for an overall response rate (ORR) of $9 \%$. Response duration was 8 and 20 months. (40). Although inclusion criteria comprised the expression of PDL1 in at least $1 \%$ of cancer or stromal cells, this apparently poor response might be related to the inclusion of 'cold' advanced DTCs or PDTCs, predicted to be resistant to ICI monotherapy. More studies are needed to clarify this point.

The role of ICIs appears to be more promising in ATCs. Apart from the evidence that ATCs present the predictive biomarkers (18), some reports from the literature have shown promising results. Kollipara and coworkers described an exceptional response with immunotherapy in a patient with $\mathrm{BRAF}^{\mathrm{V} 600 \mathrm{E}}$-positive ATC. Following 12 cycles of nivolumab, the patient presented substantial regression of tumor nodules and continued to be in complete radiographic and clinical remission 8 months after ending treatment with nivolumab (41). Similarly, Spalart and coworkers described an ATC patient showing clinical benefit after only two cycles of pembrolizumab and near complete response after three cycles (42). Recently published results obtained in 30 patients affected by ATC, included in a phase I/II trial conducted to evaluate antitumor activity of spartalizumab, another anti-PD1 antibody, as monotherapy, in ATC, showed an ORR by RECIST 1.1 (confirmed+unconfirmed partial responses (PRs)) of 5/30 (17\%) (43). Conversely, Khan and coworkers reported 2/4 PRs in ATC patients treated with pembrolizumab (44).

In summary, this limited amount of data underscores a potential role of ICI treatment in advanced thyroid carcinomas, but probably many cases of partial or complete resistance exist. Thus, use of ICIs should be rationally applied in relation to the results derived from the analysis of the full immune phenotype of the cancer or in association with other strategies aimed at overcoming ICI resistance. Furthermore, the more promising targets of ICI among thyroid carcinomas, namely ATCs, are very rare, extremely aggressive and rapidly progressive and harboring patients are mostly fragile. Thus, the therapy in this context needs to be extremely rapid, potent and characterized by acceptable toxicity. Due to the slow onset of ICI efficacy, consideration should be given to the need to associate bridging chemotherapies or targeted therapies. The need of evidence-based personalized treatment schemes and patients' eligibility criteria require the design of large well-conducted collaborative multicenter studies.

\section{Ways to overcome $\mathrm{ICI}$ resistance}

\section{Increasing tumor immunogenicity and T-cell priming}

An already approved strategy to increase T-cell priming is the combination treatment with anti-CTLA4 and anti-PD1 antibodies (9). Indeed, anti-CTLA4 treatment increases T-cell priming and CTL anti-tumor immunity and reduces Tregs in the TME. Conversely, anti-PD1 treatment enhances the T-cell effector response. This approach has been approved for melanoma with any BRAF status and renal carcinoma. A phase 2 study of Nivolumab plus Ipilimumab in RAI Refractory DTC with Exploratory Cohorts in MTC and ATC is ongoing (NCT03246958).

Chemotherapy appears to sensitize tumors to ICI increasing release of antigens and decreasing Tregs and MDSCs infiltration in the TME (9). Similarly, radiotherapy increases antigen presentation, inflammation in the TME, DC activation, release of proinflammatory cytokines and subsequently infiltration of TILs in the TME (9). Thus, the combination of ICI treatment with chemoradiotherapy might enhance the efficacy of anti-PD1 antibodies. Very recently, Chintakuntlawar and coworkers tested whether pembrolizumab might synergistically combine with chemoradiotherapy as initial ATC therapy. Unfortunately, the survival outcomes compared with historical controls were disappointing and raised uncertainty that the piloted approach merited further pursuit in ATC (45).

Cancer vaccines using DCs or tumor-specific peptides increase antigen presentation and prime $\mathrm{T}$ cells. In particular, tumor neoantigen vaccinations coupled with anti-PD1 treatment resulted in complete responses (CRs) in melanoma (46). No clinical trial of this type has been performed in thyroid carcinoma yet.

Similarly, oncolytic viruses inducing cancer cell lysis promote antigen presentation and T-cell priming (47). Thus, also their combination with ICI treatment might enhance the efficacy of anti-PD1 antibodies. No clinical trial of this type has been performed in thyroid carcinoma yet. 


\section{Overcoming T-cell exhaustion}

Following T-cell priming and migration into the tumor, the degree of T-cell activation depends on the summation of costimulatory and coinhibitory stimula. In many cases, resistance to anti-PD1 or anti-PDL1 treatments might depend on the activation of alternative inhibitory immune checkpoints other than PD1 in the $\mathrm{T}$ cell that trigger T-cell exhaustion (9). Moreover, the same inhibitory receptors might be upregulated in Tregs, where they stimulate an immunosuppressive program (9). Thus, simultaneous targeting of multiple inhibitory immune checkpoint can solve T-cell exhaustion not responsive to ICI monotherapy. Another way to amplify T-cell activation is to use costimulatory agonists that target stimulatory receptors. Combination of those agonists with ICI therapy is expected to implement the efficacy of the latter (9).

Analysis of the immune phenotype of thyroid carcinomas clearly indicated that ATCs display a very significant up-regulation of multiple inhibitory immune checkpoint receptors but also of stimulatory ones (18). Thus, combination ICI therapy or association between ICI and costimulatory agonist appears to be a promising approach to overcome T-cell exhaustion in those 'hot' cancers.

\section{Improving TME}

The main mechanisms that contribute to immunosuppression of the TME include abnormality of blood vessels, infiltration of myeloid cells, and infiltration of Tregs.

In detail, dense stromas are associated with increased mechanical forces and compression of leaky and immature neoformed blood and lymphatic vessels. These events result in hypoperfusion and the development of a hypoxic and acidic TME that supports resident and infiltrating immunosuppressive cells, induces immune checkpoint expression and facilitates the exclusion and exhaustion of CTLs (9) (Fig. 2). Thus, normalization of tumor vasculature might represent an important strategy to enhance ICI efficacy (Fig. 2). A possible way to normalize tumor vasculature foresees the use of anti-angiogenetic agents such as VEGF or VEGFR inhibitors. However, appropriate doses should be applied in order to avoid excessive loss of vasculature that is associated with exacerbation of hypoperfusion and hypoxia. Many clinical trials testing the combination of anti-angiogenetic drugs and ICI are ongoing (9). A second strategy targets the stroma with the aim to reduce the physical forces that collapse the vessels. One possible approach is represented by agents that target the renin-angiotensin axis whose inhibition reduces stromal cell activity and matrix deposition (9). Very recently, also inhibition of CXCR4 was proposed to reduce stroma deposition (9).

Combination of ICI with strategies to normalize the tumor vasculature and stroma might represent a very promising approach in PDTCs that have a dominant prevalence of altered-excluded and cold immune coordination profiles.

One strategy to reprogram the myeloid cells infiltrating the TME is to target CSF1/R. Indeed its block results in the reduction of infiltrating immunosuppressive myeloid cells and in the shift of the remaining ones toward a T-cell-stimulating phenotype (48) (Fig. 2). This approach is supposed to be very efficient in those advanced thyroid carcinomas, specially ATCs, that are richly infiltrated by TAMs (21). Similarly, VEGF inhibition blocks the infiltration of MDSCs and pro-tumoral TAMs and increases mature DCs (49).

Clinical trials that foresee the combination of ICI with CSF1/R have not been conducted in thyroid carcinoma yet.

A hypoxic TME triggers enrichment in Tregs that strongly inhibit APCs and CTLs (49, 50). Moreover, Tregs appears to have a superior capacity than CTLs themselves to recognize antigens, possibly promoting further immunosuppression when antigen presentation increases or when vaccinations are applied (50). Thus, Tregs reprogramming is instrumental for the success of immunotherapies (Fig. 2), although it should be kept in mind that their absence increases immune-related adverse events $(49,50)$.

Interestingly, treatment with anti-CTLA4 antibodies is also able to inhibit CTLA4-expressing Tregs $(49,50)$. On the contrary, treatment with anti-PD1 antibodies, by blocking PD1 expressed on Tregs surface, activates their immunosuppressive function and may contribute to disease hyperprogression during therapy (51).

Treg depletion by targeting cell surface molecules such as CD25 or CCR4 resulted in reduction of Tregs in the TME but in mixed effects in CTL function (49). Conversely, Treg reprogramming by modulating CD25 or OX40 or GITR or 4-1BB or IDO signaling axes resulted in the inhibition of the immunosuppressive function and the conversion to an immunostimulatory effector phenotype (49). VEGF pathway inhibition is also associated with reduced immune checkpoint expression and Treg infiltration (9).

Treg reprogramming might have its relevance in thyroid carcinomas due to the high representation of these cells specially in advanced cancers $(22,23,24)$. The 
Low infiltrated cancers

Cold or immunoexcluded

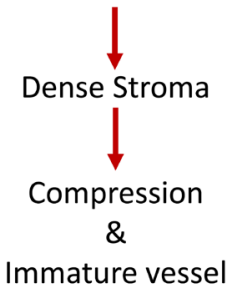

Normalization of vasculature

\section{A. $\alpha$ VEGF/VEGFR \\ B. Renin/Angiotensin Axis \\ C. $\alpha$ CXCR4}

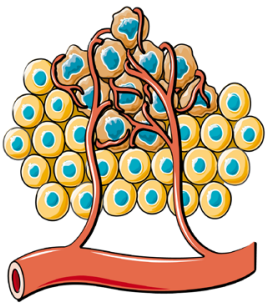

Hypoxia \& Acid TME

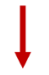

个Immunosuppressant cells $\downarrow$ CTL cells
High infiltrated cancers Hot or immunosuppressed

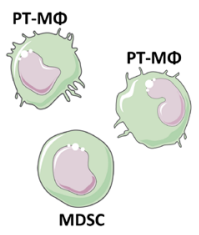
Reprogramming
Myeloid cells

A. CFS1/R

B. $\alpha$ VEGF/VEGFR

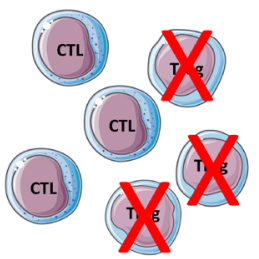

A. $\alpha$ CTLA4

B. $\mathrm{CD} 25+$

CCR

or OX40 or GITR or 4-IBB

or IDO

\section{Figure 2}

Therapeutic approaches to reverse the immunosuppression of the TME. Left: Tumor with a dense stroma are generally characterized by low leucocyte infiltration (cold or immunoexcluded phenotypes). In these cancers, the presence of compression forces and immature vessels results in hypoperfusion and in the development of a hypoxic and acidic TME that supports resident and infiltrating immunosuppressive cells, induces immune checkpoint expression and facilitates the exclusion and exhaustion of CTLS. An effective strategy to enhance ICl efficacy may consist in the normalization of tumor vasculature targeting different pathways. Right: Treatment of tumors with high leucocytes infiltration (hot or immunosuppressed phenotypes) could take advantage from therapies that promote a reprogramming of the myeloid cells including a shift of macrophages to the antitumoral phenotype or the recruitment of functional DCs. Moreover, Treg reprogramming inhibits their immunosuppressive function and favors their conversion to an immunostimulatory effector phenotype. РТ-МФ: pro-tumoral macrophages; AT-MФ: anti-tumoral macrophages.

evidence of a correlation between IDO1 expression and Treg density in PTC TME (33) suggested the possibility that IDO1 inhibition might represent a valid support for ICI treatment in order to enhance its efficacy in those cancers. Similarly, the evidence of cancers with high expression of stimulatory immune checkpoint, such as ATCs (18), suggested the possibility to disrupt the suppressive function of Tregs by exploiting these receptors in combination with ICIs.

\section{Combination with targeted therapies}

Summarizing what is already described in the former paragraph, combination of ICI with anti-angiogenetic drugs appears very promising for the treatment of cancer because it enhances CTL infiltration and activity, reduces CTL dysfunction, reduces suppressive function of Tregs and MDSCs, and increases density of APCs (i.e. DCs) (9). All those effects result in increased tumor control, reduced metastases and enhanced survival, as already demonstrated by the results of several clinical trials. Just to mention the most significant, very recently, a phase III clinical trial demonstrated an improvement in PFS and OS in NSCLC patients treated with atezolizumab and chemotherapy when also the anti-VEGF antibody bevacizumab was added (52). The results were independent from the mutational status of the tumors and received rapid FDA approval as first-line treatment for EGFR and ALK mutation-negative NSCLCs. In ATCs and advanced PDTCs, a phase II clinical trial is ongoing that foresees in BRAF and RAS-negative 
patients the combination of atezolizumab, chemotherapy and bevacizumab (NCT03181100).

Combination of anti-PD1/PDL1 treatment with BRAF and/or MEK inhibitors was associated with expansion of CTLs, avoidance of exhaustion and apoptosis, and enhanced anti-tumor immunity (53).

Parangi and coworkers utilized an immunocompetent orthotopic murine model of ATC to assess the combination of BRAF ${ }^{\mathrm{V} 600 \mathrm{E}}$ inhibitor PLX4720 and antiPDL1 or anti-PD1 antibody on survival. Combination therapy dramatically improved mouse survival. Maximal tumour reduction was associated with increases in the number and cytotoxicity of CD8+ T cells and NK cells, as well as increases in anti-tumoral TAMs and decreases in myeloid-derived suppressor-like cells (54). Indeed, a recent report by Antonello et al. showed that treatment of diploid BRAF ${ }^{\mathrm{V} 600 \mathrm{E}}$ papillary thyroid carcinoma cells with vemurafenib induced the outgrowth of vemurafenib-resistant subclones containing additional passenger mutation (amplification of chromosome 5 and de novo mutations in the RBM (RNA-binding motifs) genes family (i.e. RBMX, RBM10)), that responded to combined therapy with vemurafenib and the CDK4/6 inhibitor palpociclib, but that were also expected to be more immunogenic than the cells in the original culture (55). Parangi and coworkers also tested the immunocompetent orthotopic murine model of ATC with a combination of lenvatinib and anti-PD1 or antiPDL1 antibodies and demonstrated that lenvatinib (a TKI that targets VEGFR1, 2, and 3, Platelet-derived growth factor receptor $\alpha$, Fibroblast growth factor receptor, and the KIT and RET tyrosine kinases) led to significant tumor shrinkage and increased survival, while combination therapy led to dramatic improvements in both. Lenvatinib monotherapy increased tumorinfiltrating macrophages, CD8+ T cells, Tregs and, most notably, polymorphonuclear MDSCs (PMN-MDSCs). While both combination therapies (anti-PD1 or antiPDL1 antibodies + vemurafenib or lenvatinib) led to further increases in CD8+ T cells, only the lenvatinib and anti-PD1 combination decreased PMN-MDSCs. Thus, this latter combination appears to hold the better pharmacodynamics profile (56).

Furthermore, salvage pembrolizumab added at failure to TKIs (lenvatinib or dabrafenib plus trametinib) in the treatment of 12 ATC patients showed PR in 5/12 (42\%), stable disease in $4 / 12$ (33\%), progressive disease in $3 / 12$ (25\%), with a median OS of 10.4 months but a wide range (95\% CI: 1.7-12.15; range: 5.4-40 months) indicating some long-lasting responders (57).

\section{Epigenetic modulation}

In cancer, down-regulation of immunostimulatory cytokines or of antigen presentation may occur via the epigenetic modulation of specific genes. Thus, DNA methyltransferase and histone deacetylase inhibitors might be used to gain immunogenicity and implement ICI efficacy. Multiple clinical trials are exploring the feasibility of this strategy in several cancer types (58). However, no clinical trial of this type has been performed in thyroid carcinoma yet.

\section{Enhancing tumor infiltration by T cells}

Adoptive cell transfer includes injection to the cancer patient of expanded tumor-specific TILs, TCR-engineered lymphocytes, chimeric antigen receptor (CAR)-T cells or endogenous $\mathrm{T}$ cells (Recently reviewed in (59)). This type of approach may allow a rich colonization of the cancer by highly tumor-reactive $\mathrm{T}$ cells whose anti-cancer action can be magnified by contemporary ICI treatment (9). No clinical trial of this type has been performed in thyroid carcinoma yet.

\section{Modulation of gut microbiota}

The microbiome has been implicated in tumorigenesis as well as activation or suppression of the immune system that can contribute to tumor control or escape. In regards to ICI efficacy, a growing body of evidence indicates that the gut microbiota may enhance the antitumor immune response (60). Several gut bacteria such as Akkermansia muciniphila, Bifidobacterium spp., Faecalibacterium spp., and Bacteroides fragilis have been shown to display strong regulatory effects on ICI treatments (61). Thus, diet modifications, that is, increased fiber intake with its immunostimulatory action, or probiotics administration, that is, Bifidobacterium cocktail that increases DC activation, have been proposed to enhance ICI efficacy (9). No clinical trial of this type has been performed in thyroid carcinoma yet.

\section{Ongoing clinical trials}

Table 1 summarizes the ongoing clinical trials specifically designed for thyroid carcinoma with the anti-PD1 agents pembrolizumab and nivolumab and the anti-PDL1 agents atezolizumab and durvalumab (from the ClinicalTrials. gov. site, last assessment on February $19^{\text {th }}$ 2020). 


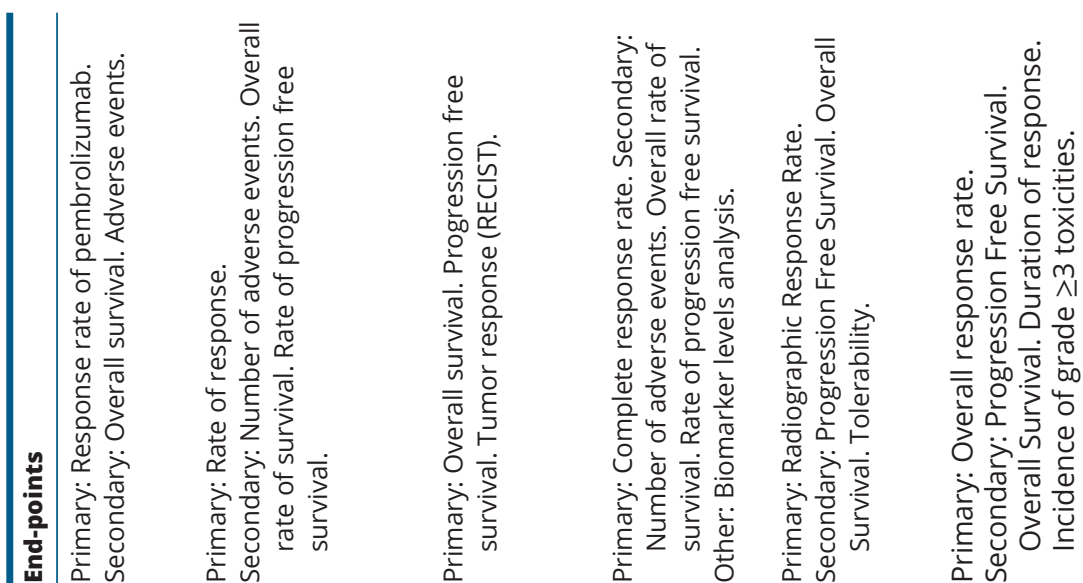

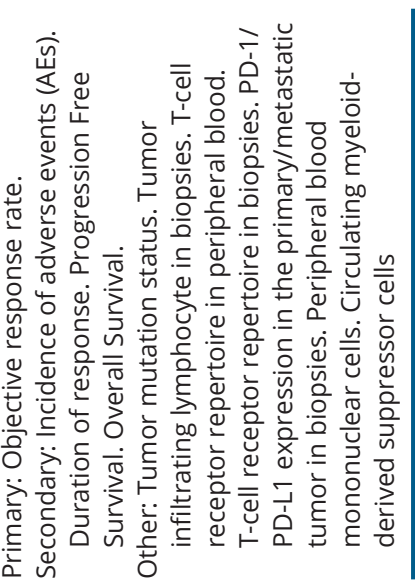

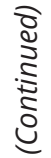
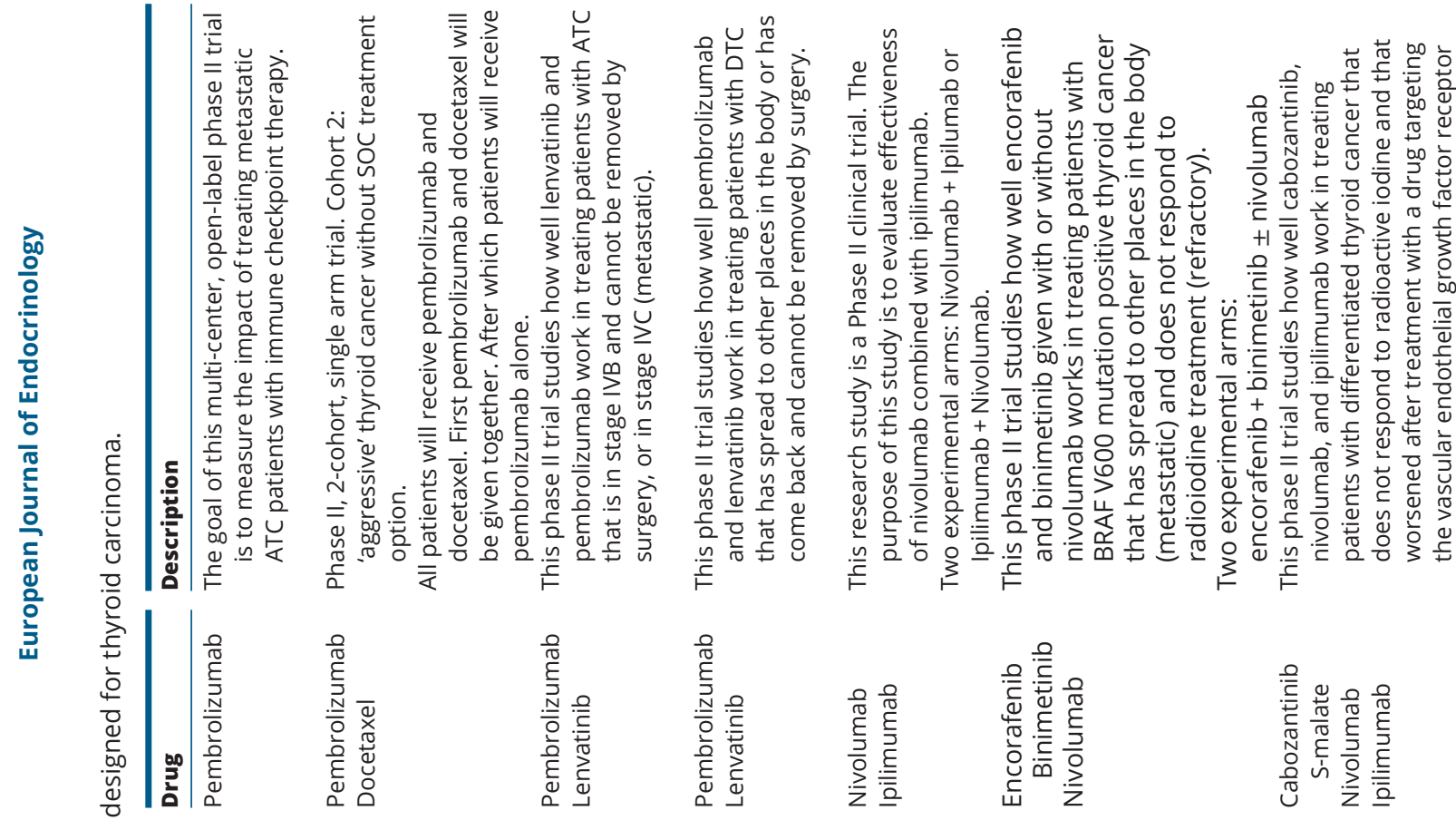

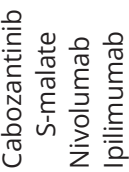
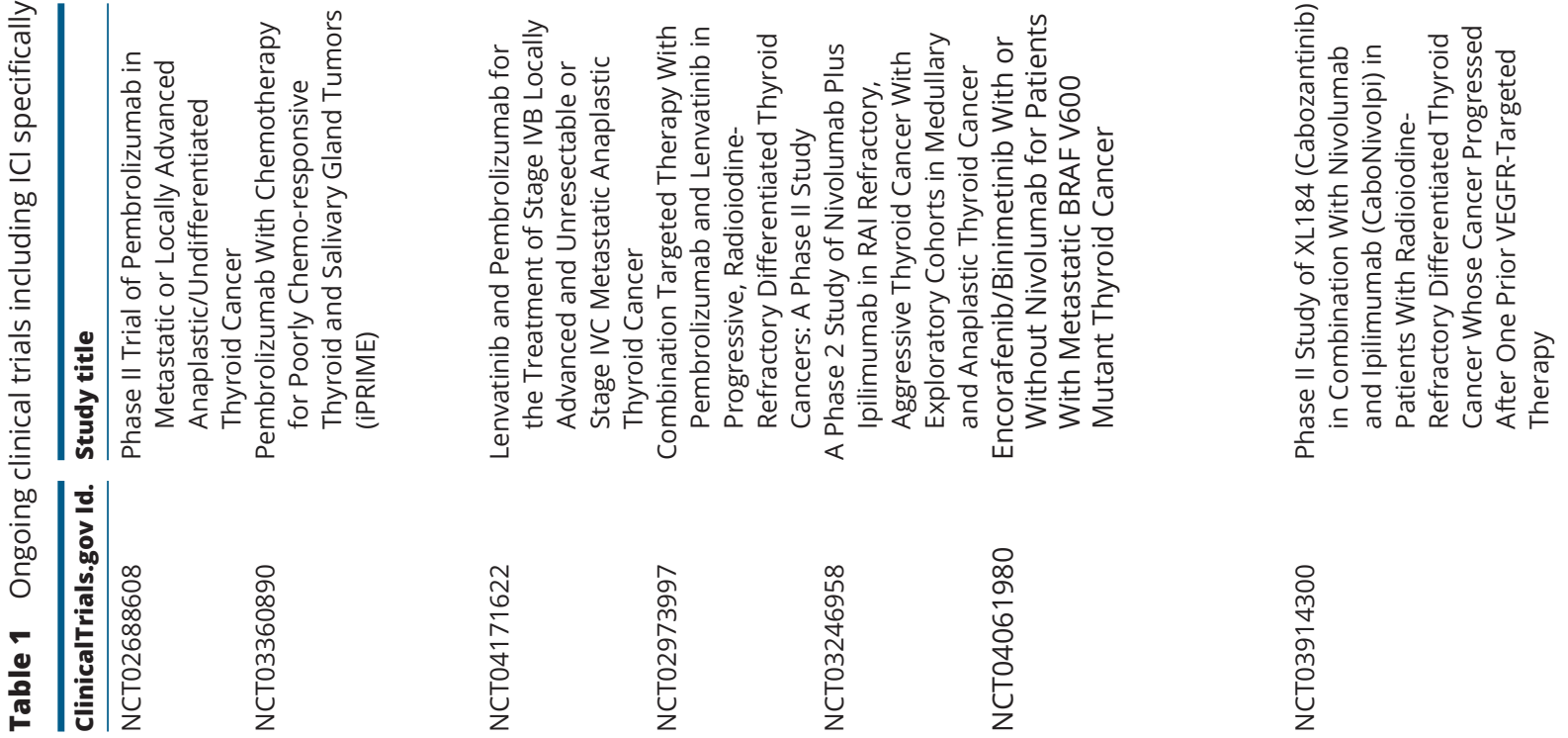

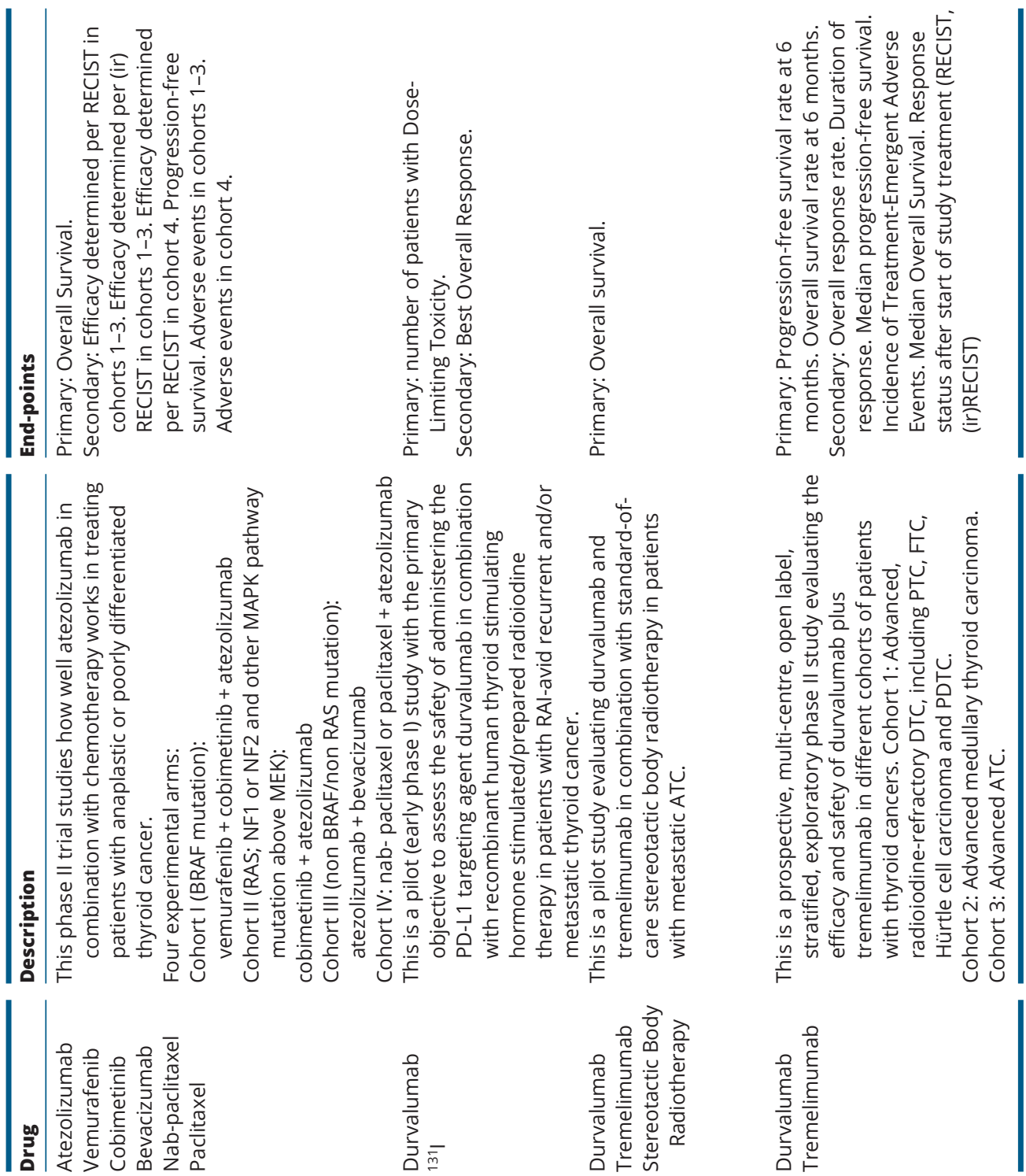

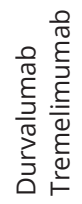
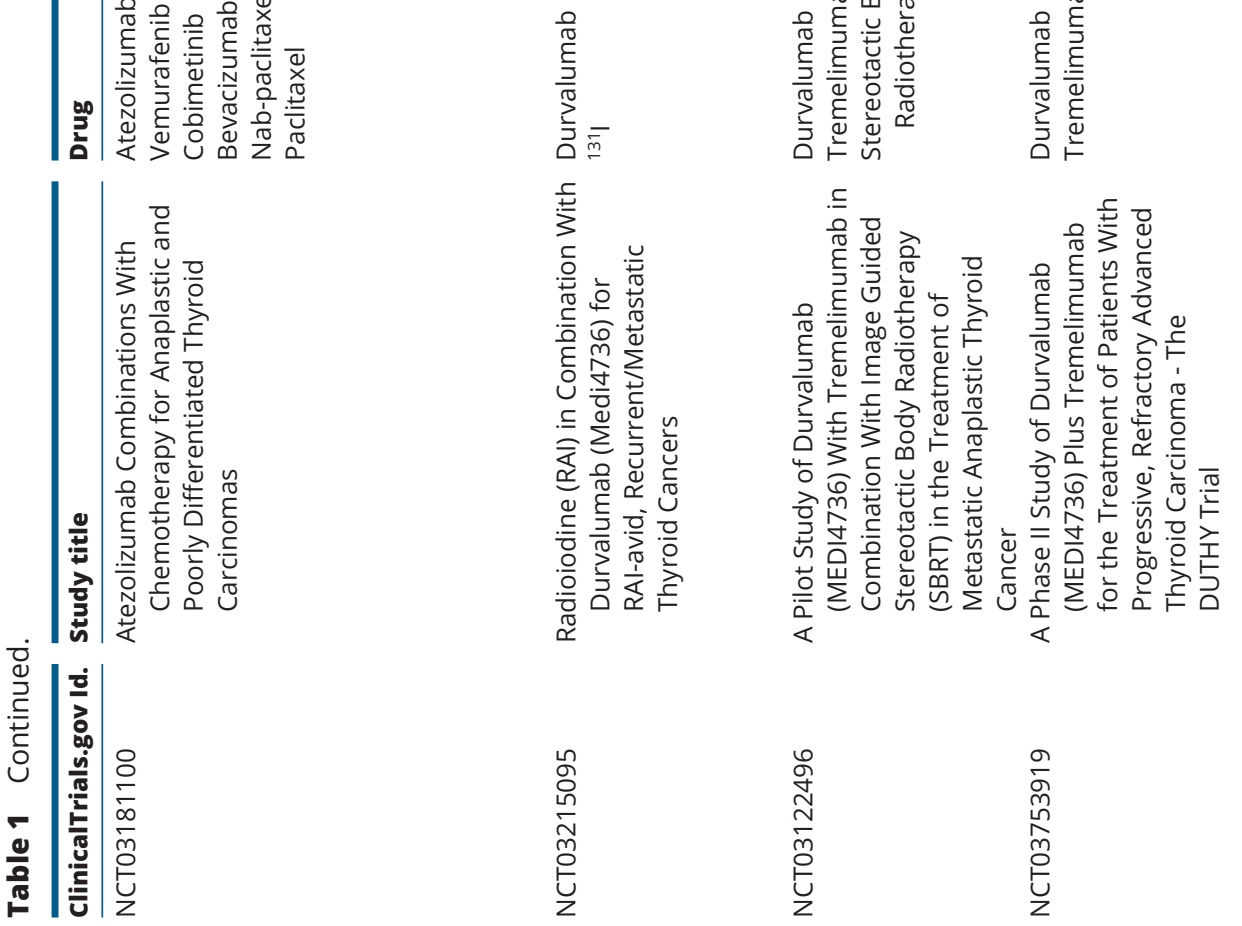


\section{Future directions}

ICIs are emerging as possible novel therapeutic tools also for advanced thyroid carcinomas. The ongoing clinical trials, the widespread treatment of single cases outside of clinical trials, a deeper characterization of the immune phenotype of the treated cancers, the detection of novel efficacy biomarkers, and the integration of all the obtained data will certainly produce rationale guidelines for the use of these drugs as single agents or in combination with chemotherapy, radiotherapy, or targeted therapies.

The recent evidence that TAMs, when appropriately stimulated, have the capacity to cooperate with T cells for an anti-tumoral action opens the road to combination therapies targeting simultaneously both T cells and TAMs, specially in ATC. In particular, T-cell-focused treatments with ICIs would greatly benefit from the combination with activators of proimmune TAM activities such as CD40 agonists, STING activators or other of IFN $\alpha$ inducers and with inhibitors of pro-tumoral TAM functions, that is, inhibitors of TGF $\beta$ or class IIa Histone Deacetylase (62).

Furthermore, although at present time ICIs represent the real novel revolution in oncology, also other anticancer immunotherapeutic strategies are in the phase of being implemented, including those based on adoptive cell transfer or personalized vaccination. In particular, endogenous T-cell therapy, based on the isolation and expansion of antigen-specific $\mathrm{T}$ cells from blood, is very promising due the potential high efficacy and low toxicity. We expect that also those novel approaches will find a space in the treatment of advanced thyroid carcinomas, eventually in combination with ICIs.

\section{Declaration of interest}

The authors declare that there is no conflict of interest that could be perceived as prejudicing the impartiality of this review.

\section{Funding}

This research did not receive any specific grant from any funding agency in the public, commercial or not-for-profit sector.

\section{Acknowledgements}

The figures were designed using elements from Servier Medical Art by Servier that are licensed under a Creative Commons Attribution 3.0 Unported License.

\section{References}

1 Hanahan D \& Weinberg RA. Hallmarks of cancer: the next generation. Cell 2011144 646-674. (https://doi.org/10.1016/j.cell.2011.02.013)
2 Rock KL, Reits E \& Neefjes J. Present yourself! By MHC class I and MHC class II molecules. Trends in Immunology 201637 724-737. (https://doi.org/10.1016/j.it.2016.08.010)

3 Prendergast GC. Immune escape as a fundamental trait of cancer: focus on IDO. Oncogene 200827 3889-3900. (https://doi. org/10.1038/onc.2008.35)

4 Coley WB. The treatment of malignant tumors by repeated inoculations of erysipelas. With a report of ten original cases. 1893. Clinical Orthopaedics and Related Research 1991262 3-11.

5 Krummel MF \& Allison JP. CD28 and CTLA-4 have opposing effects on the response of T cells to stimulation. Journal of Experimental Medicine 1995182 459-465. (https://doi.org/10.1084/ jem.182.2.459)

6 Leach DR, Krummel MF \& Allison JP. Enhancement of antitumor immunity by CTLA-4 blockade. Science 1996271 1734-1736. (https://doi.org/10.1126/science.271.5256.1734)

7 Keir ME, Butte MJ, Freeman GJ \& Sharpe AH. PD-1 and its ligands in tolerance and immunity. Annual Review of Immunology 200826 677-704. (https://doi.org/10.1146/annurev. immunol.26.021607.090331)

8 Topalian SL, Drake CG \& Pardoll DM. Targeting the PD-1/ B7-H1(PD-L1) pathway to activate anti-tumor immunity. Current Opinion in Immunology 201224 207-212. (https://doi.org/10.1016/j. coi.2011.12.009)

9 Fares CM, Van Allen EM, Drake CG, Allison JP \& Hu-Lieskovan S. Mechanisms of resistance to immune checkpoint blockade: why does checkpoint inhibitor immunotherapy not work for all patients? American Society of Clinical Oncology Educational Book 201939 $147-164$.

10 Schlumberger M, Brose M, Elisei R, Leboulleux S, Luster M, Pitoia F \& Pacini F. Definition and management of radioactive iodine-refractory differentiated thyroid cancer. Lancet: Diabetes and Endocrinology 20142 356-358. (https://doi.org/10.1016/S22138587(13)70215-8)

11 Viola D, Valerio L, Molinaro E, Agate L, Bottici V, Biagini A, Lorusso L, Cappagli V, Pieruzzi L, Giani C et al. Treatment of advanced thyroid cancer with targeted therapies: ten years of experience. Endocrine-Related Cancer 201623 R185-R205. (https:// doi.org/10.1530/ERC-15-0555)

12 Schumacher TN \& Schreiber RD. Neoantigens in cancer immunotherapy. Science 2015348 69-74. (https://doi.org/10.1126/ science.aaa4971)

13 Le DT, Durham JN, Smith KN, Wang H, Bartlett BR, Aulakh LK, Lu S, Kemberling H, Wilt C, Luber BS et al. Mismatch repair deficiency predicts response of solid tumors to PD-1 blockade. Science $2017 \mathbf{3 5 7}$ 409-413. (https://doi.org/10.1126/science.aan6733)

14 Spranger S, Bao R \& Gajewski TF. Melanoma-intrinsic $\beta$-catenin signalling prevents anti-tumour immunity. Nature 2015523 231-235. (https://doi.org/10.1038/nature14404)

15 Vatner RE \& Janssen EM. STING, DCs and the link between innate and adaptive tumor immunity. Molecular Immunology 2019110 13-23. (https://doi.org/10.1016/j.molimm.2017.12.001)

16 Angell TE, Lechner MG, Jang JK, LoPresti JS \& Epstein AL. MHC class I loss is a frequent mechanism of immune escape in papillary thyroid cancer that is reversed by interferon and selumetinib treatment in vitro. Clinical Cancer Research 201420 6034-6044. (https://doi. org/10.1158/1078-0432.CCR-14-0879)

17 Ulisse S, Tuccilli C, Sorrenti S, Antonelli A, Fallahi P, D'Armiento E, Catania A, Tartaglia F, Amabile MI, Giacomelli L et al. PD-1 ligand expression in epithelial thyroid cancers: potential clinical implications. International Journal of Molecular Sciences 201920 E1405. (https://doi.org/10.3390/ijms20061405)

18 Giannini R, Moretti S, Ugolini C, Macerola E, Menicali E, Nucci N, Morelli S, Colella R, Mandarano M, Sidoni A et al. Immune profiling of thyroid carcinomas suggests the existence of two major phenotypes: an ATC-like and a PDTC-like. Journal of Clinical 
Endocrinology and Metabolism 2019104 3557-3575. (https://doi. org/10.1210/jc.2018-01167)

19 Galon J \& Bruni D. Approaches to treat immune hot, altered and cold tumours with combination immunotherapies. Nature Reviews: Drug Discovery 201918 197-218. (https://doi.org/10.1038/s41573018-0007-y)

20 Spranger S \& Gajewski TF. Tumor-intrinsic oncogene pathways mediating immune avoidance. Oncoimmunology 20165 e1086862. (https://doi.org/10.1080/2162402X.2015.1086862)

21 Ferrari SM, Fallahi P, Galdiero MR, Ruffilli I, Elia G, Ragusa F, Paparo SR, Patrizio A, Mazzi V, Varricchi G et al. Immune and inflammatory cells in thyroid cancer microenvironment. International Journal of Molecular Sciences 201920 4413. (https://doi.org/10.3390/ ijms20184413)

22 French JD, Weber ZJ, Fretwell DL, Said S, Klopper JP \& Haugen BR. Tumor-associated lymphocytes and increased FoxP3+ regulatory $\mathrm{T}$ cell frequency correlate with more aggressive papillary thyroid cancer. Journal of Clinical Endocrinology and Metabolism 201095 2325-2333. (https://doi.org/10.1210/jc.2009-2564)

23 French JD, Kotnis GR, Said S, Raeburn CD, McIntyre Jr RC, Klopper JP \& Haugen BR. Programmed death- $1+$ T cells and regulatory $\mathrm{T}$ cells are enriched in tumor-involved lymph nodes and associated with aggressive features in papillary thyroid cancer. Journal of Clinical Endocrinology and Metabolism 201297 E934-E943. (https:// doi.org/10.1210/jc.2011-3428)

24 Severson JJ, Serracino HS, Mateescu V, Raeburn CD, McIntyre Jr RC, Sams SB, Haugen BR \& French JD. PD-1+Tim-3+ CD8+ T lymphocytes display varied degrees of functional exhaustion in patients with regionally metastatic differentiated thyroid cancer. Cancer Immunology Research 20153 620-630. (https://doi.org/10.1158/23266066.CIR-14-0201)

25 Caillou B, Talbot M, Weyemi U, Pioche-Durieu C, Al Ghuzlan A, Bidart JM, Chouaib S, Schlumberger M \& Dupuy C. Tumor-associated macrophages (TAMs) form an interconnected cellular supportive network in anaplastic thyroid carcinoma. PLoS ONE 20116 e22567. (https://doi.org/10.1371/journal.pone.0022567)

26 Liu C, Peng W, Xu C, Lou Y, Zhang M, Wargo JA, Chen JQ, Li HS, Watowich SS, Yang Y et al. BRAF inhibition increases tumor infiltration by $\mathrm{T}$ cells and enhances the antitumor activity of adoptive immunotherapy in mice. Clinical Cancer Research 201319 393-403. (https://doi.org/10.1158/1078-0432.CCR-12-1626)

27 Peng W, Chen JQ, Liu C, Malu S, Creasy C, Tetzlaff MT, Xu C, McKenzie JA, Zhang C, Liang X et al. Loss of PTEN promotes resistance to T cell-mediated immunotherapy. Cancer Discovery 2016 6 202-216. (https://doi.org/10.1158/2159-8290.CD-15-0283)

28 Ryder M, Gild M, Hohl TM, Pamer E, Knauf J, Ghossein R, Joyce JA $\&$ Fagin JA. Genetic and pharmacological targeting of CSF-1/CSF-1R inhibits tumor-associated macrophages and impairs BRAF-induced thyroid cancer progression. PLOS ONE 20138 e54302. (https://doi. org/10.1371/journal.pone.0054302)

29 Puxeddu E, Knauf JA, Sartor MA, Mitsutake N, Smith EP, Medvedovic M, Tomlinson CR, Moretti S \& Fagin JA. RET/PTCinduced gene expression in thyroid PCCL3 cells reveals early activation of genes involved in regulation of the immune response. Endocrine-Related Cancer 200512 319-334. (https://doi.org/10.1677/ erc.1.00947)

30 Borrello MG, Alberti L, Fischer A, Degl'innocenti D, Ferrario C, Gariboldi M, Marchesi F, Allavena P, Greco A, Collini P et al. Induction of a proinflammatory program in normal human thyrocytes by the RET/PTC1 oncogene. PNAS 2005102 14825-14830. (https://doi.org/10.1073/pnas.0503039102)

31 Wang J, Knauf JA, Basu S, Puxeddu E, Kuroda H, Santoro M, Fusco A $\&$ Fagin JA. Conditional expression of RET/PTC induces a weak oncogenic drive in thyroid pccl3 cells and inhibits thyrotropin action at multiple levels. Molecular Endocrinology 200317 1425-1436. (https://doi.org/10.1210/me.2003-0041)
32 Prieur A \& Peeper DS. Cellular senescence in vivo: a barrier to tumorigenesis. Current Opinion in Cell Biology 200820 150-155. (https://doi.org/10.1016/j.ceb.2008.01.007)

33 Moretti S, Menicali E, Voce P, Morelli S, Cantarelli S, Sponziello M, Colella R, Fallarino F, Orabona C, Alunno A et al. Indoleamine 2,3-dioxygenase 1 (IDO1) is up-regulated in thyroid carcinoma and drives the development of an immunosuppressant tumor microenvironment. Journal of Clinical Endocrinology and Metabolism 201499 E832-E840. (https://doi.org/10.1210/jc.2013-3351)

34 Moretti S, Menicali E, Nucci N, Voce P, Colella R, Melillo RM, Liotti F, Morelli S, Fallarino F, Macchiarulo A et al. Signal transducer and activator of transcription 1 plays a pivotal role in RET/PTC3 oncogene-induced expression of indoleamine 2,3-dioxygenase 1. Journal of Biological Chemistry 2017292 1785-1797. (https://doi. org/10.1074/jbc.M116.745448)

35 Moretti S, Nucci N, Menicali E, Morelli S, Bini V, Colella R, Mandarano M, Sidoni A \& Puxeddu E. The aryl hydrocarbon receptor is expressed in thyroid carcinoma and appears to mediate epithelialmesenchymal-transition. Cancers 202012 E145. (https://doi. org/10.3390/cancers12010145)

36 Occhi G, Barollo S, Regazzo D, Bertazza L, Galuppini F, Guzzardo V, Jaffrain-Rea ML, Vianello F, Ciato D, Ceccato F et al. A constitutive active MAPK/ERK pathway due to BRAFV660E positively regulates AHR pathway in PTC. Oncotarget 20156 32104-32114. (https://doi. org/10.18632/oncotarget.5194)

37 Na KJ \& Choi H. Immune landscape of papillary thyroid cancer and immunotherapeutic implications. Endocrine-Related Cancer 201825 523-531. (https://doi.org/10.1530/ERC-17-0532)

38 Popovic A, Jaffee EM \& Zaidi N. Emerging strategies for combination checkpoint modulators in cancer immunotherapy. Journal of Clinical Investigation 2018128 3209-3218. (https://doi.org/10.1172/ JCI120775)

39 Tumeh PC, Harview CL, Yearley JH, Shintaku IP, Taylor EJ, Robert L, Chmielowski B, Spasic M, Henry G, Ciobanu V et al. PD-1 blockade induces responses by inhibiting adaptive immune resistance. Nature 2014515 568-571. (https://doi.org/10.1038/nature13954)

40 Mehnert JM, Varga A, Brose MS, Aggarwal RR, Lin CC, Prawira A, de Braud F, Tamura K, Doi T, Piha-Paul SA et al. Safety and antitumor activity of the anti-PD-1 antibody pembrolizumab in patients with advanced, PD-L1-positive papillary or follicular thyroid cancer. $B M C$ Cancer 201919 196. (https://doi.org/10.1186/s12885-019-5380-3)

41 Kollipara R, Schneider B, Radovich M, Babu S \& Kiel PJ. Exceptional response with immunotherapy in a patient with anaplastic thyroid cancer. Oncologist 201722 1149-1151. (https://doi.org/10.1634/ theoncologist.2017-0096)

42 Spalart V, Legius B, Segers K, Coolen J, Maes B \& Decoster L. Dramatic response to first line single agent pembrolizumab in anaplastic thyroid carcinoma. Case Reports in Endocrinology 201920199095753. (https://doi.org/10.1155/2019/9095753)

43 Wirth LJ, Eigendorff E, Capdevila J, Paz-Ares LG, Lin CC, Taylor MH, Ramlau R, Butler M, Delord JP, Horvath Z et al. Phase I/II study of spartalizumab (PDR001), an anti-PD1 $\mathrm{mAb}$, in patients with anaplastic thyroid cancer. Journal of Clinical Oncology 201836 (Supplement 15) 6024-6024. (https://doi.org/10.1200/JCO.2018.36.15_suppl.6024)

44 Khan SA, Kurian P, Mobley B, Burks T, Beg MS, Ross JS, Ali SM \& Bowles DW. Relationship of anaplastic thyroid cancer high tumor mutation burden and MSI-H status with response to anti-PD1 monotherapy. Journal of Clinical Oncology 201836 (Supplement 15) e18114. (https://doi.org/10.1200/JCO.2018.36.15_suppl.e18114)

45 Chintakuntlawar AV, Yin J, Foote RL, Kasperbauer JL, Rivera M, Asmus E, Garces NI, Janus JR, Liu M, Ma DJ et al. A Phase 2 study of pembrolizumab combined with chemoradiotherapy as initial treatment for anaplastic thyroid cancer. Thyroid 201929 1615-1622. (https://doi.org/10.1089/thy.2019.0086)

46 Ott PA, Hu Z, Keskin DB, Shukla SA, Sun J, Bozym DJ, Zhang W, Luoma A, Giobbie-Hurder A, Peter L et al. An immunogenic personal 
neoantigen vaccine for patients with melanoma. Nature $2017 \mathbf{5 4 7}$ 217-221. (https://doi.org/10.1038/nature22991)

47 Guo ZS, Liu Z \& Bartlett DL. Oncolytic immunotherapy: dying the right way is a key to eliciting potent antitumor immunity. Frontiers in Oncology 20144 74. (https://doi.org/10.3389/fonc.2014.00074)

48 Ruffell B \& Coussens LM. Macrophages and therapeutic resistance in cancer. Cancer Cell 201527 462-472. (https://doi.org/10.1016/j. ccell.2015.02.015)

49 Shitara K \& Nishikawa H. Regulatory T cells: a potential target in cancer immunotherapy. Annals of the New York Academy of Sciences 20181417 104-115. (https://doi.org/10.1111/nyas.13625)

50 Nishikawa H \& Sakaguchi S. Regulatory T cells in cancer immunotherapy. Current Opinion in Immunology 201427 1-7. (https://doi.org/10.1016/j.coi.2013.12.005)

51 Togashi Y, Shitara K \& Nishikawa H. Regulatory T cells in cancer immunosuppression - implications for anticancer therapy. Nature Reviews: Clinical Oncology 201916 356-371. (https://doi.org/10.1038/ s41571-019-0175-7)

52 Socinski MA, Jotte RM, Cappuzzo F, Orlandi F, Stroyakovskiy D, Nogami N, Rodríguez-Abreu D, Moro-Sibilot D, Thomas CA, Barlesi F et al. Atezolizumab for first-line treatment of metastatic nonsquamous NSCLC. New England Journal of Medicine 2018378 2288-2301. (https://doi.org/10.1056/NEJMoa1716948)

53 Ebert PJR, Cheung J, Yang Y, McNamara E, Hong R, Moskalenko M, Gould SE, Maecker H, Irving BA, Kim JM et al. MAP kinase inhibition promotes $\mathrm{T}$ cell and anti-tumor activity in combination with PD-L1 checkpoint blockade. Immunity 201644 609-621. (https://doi. org/10.1016/j.immuni.2016.01.024)

54 Gunda V, Gigliotti B, Ndishabandi D, Ashry T, McCarthy M, Zhou Z, Amin S, Freeman GJ, Alessandrini A \& Parangi S. Combinations of BRAF inhibitor and anti-PD-1/PD-L1 antibody improve survival and tumour immunity in an immunocompetent model of orthotopic murine anaplastic thyroid cancer. British Journal of Cancer 2018119 1223-1232. (https://doi.org/10.1038/s41416-018-0296-2)

55 Antonello ZA, Hsu N, Bhasin M, Roti G, Joshi M, Van Hummelen P, Ye E, Lo AS, Karumanchi SA, Bryke CR et al. Vemurafenib-resistance via de novo RBM genes mutations and chromosome 5 aberrations is overcome by combined therapy with palbociclib in thyroid carcinoma with BRAFV600E. Oncotarget 20178 84743-84760. (https://doi.org/10.18632/oncotarget.21262)

56 Gunda V, Gigliotti B, Ashry T, Ndishabandi D, McCarthy M, Zhou Z, Amin S, Lee KE, Stork T, Wirth L et al. Anti-PD-1/PD-L1 therapy augments lenvatinib's efficacy by favorably altering the immune microenvironment of murine anaplastic thyroid cancer. International Journal of Cancer 2019144 2266-2278. (https://doi.org/10.1002/ ijc.32041)

57 Iyer PC, Dadu R, Gule-Monroe M, Busaidy NL, Ferrarotto R, Habra MA, Zafereo M, Williams MD, Gunn GB, Grosu H et al. Salvage pembrolizumab added to kinase inhibitor therapy for the treatment of anaplastic thyroid carcinoma. Journal for ImmunoTherapy of Cancer 20186 68. (https://doi.org/10.1186/ s40425-018-0378-y)

58 Mazzone R, Zwergel C, Mai A \& Valente S. Epi-drugs in combination with immunotherapy: a new avenue to improve anticancer efficacy. Clinical Epigenetics 20179 59. (https://doi.org/10.1186/s13148-0170358-y)

59 Ott PA, Dotti G, Yee C \& Goff SL. An update on adoptive T-cell therapy and neoantigen vaccines. American Society of Clinical Oncology Educational Book 201939 e70-e78. (https://doi.org/10.1200/ EDBK_238001)

60 Gong J, Chehrazi-Raffle A, Placencio-Hickok V, Guan M, Hendifar A $\&$ Salgia R. The gut microbiome and response to immune checkpoint inhibitors: preclinical and clinical strategies. Clinical and Translational Medicine 20198 9. (https://doi.org/10.1186/s40169-019-0225-x)

61 Salek Farrokhi A, Darabi N, Yousefi B, Askandar RH, Shariati M \& Eslami M. Is it true that gut microbiota is considered as panacea in cancer therapy? Journal of Cellular Physiology 2019234 14941-14950. (https://doi.org/10.1002/jcp.28333)

62 Bercovici N, Guérin MV, Trautmann A \& Donnadieu E. The remarkable plasticity of macrophages: a chance to fight cancer. Frontiers in Immunology 201910 1563. (https://doi.org/10.3389/ fimmu.2019.01563)

Received 1 April 2020

Revised version received 5 May 2020

Accepted 20 May 2020 